\title{
Intractable pain treated with intrathecal isotonic iced saline
}

\author{
MARTIN H. SAVITZ AND LEONARD I. MALIS
}

From the Department of Neurosurgery, Mount Sinai Hospital, New York, U.S.A.

SUMMARY Hitchcock's original method of hypothermic subarachnoid irrigation employed both temperature and osmolarity. Spinal cooling was then abandoned in favour of intrathecal injection of normothermic hypertonic saline. Modifications of the procedure that followed have continued to accept hyperosmolarity as the factor causing pain relief. Fifty patients were treated by a technique evolved to enhance the effect of hypothermia while avoiding the complications associated with hyperosmolar solutions. For the cases of terminal carcinoma and others considered to be poor surgical risks, the results have been quite satisfactory. For non-neoplastic painful syndromes, rapid perfusion cooling of the subarachnoid space offers an alternative therapeutic approach.

The effects of hypothermia on the nervous system (Denny-Brown, Adams, and Brenner, 1945) and perfusion of the subarachnoid space (Albin, White, and Donald, 1961) have been studied for many years. Hitchcock $(1967 \mathrm{a}, \mathrm{b})$ introduced subarachnoid irrigation with cold solutions for the relief of intractable pain and subsequently inquired into the hyperosmolarity of his solution. Ensuing clinical research explored the use of anaesthesia (Nicholson and Roberts, 1968; Collins, Juras, and Van Houten, 1969), varying sites of injection (Hitchcock, 1969; Mathews, Ambruso, and Osterholm, 1970), prolonged irrigation (Tsubokawa, 1969a), increasingly hypertonic saline (Hitchcock, 1970), and elaborate perfusion apparatus (Negrin, 1970). To our knowledge, no previous investigators have reported the primary results of brief intrathecal injection of isotonic iced saline.

\section{METHOD}

The irrigating solution was prepared by keeping $500 \mathrm{ml}$. pour bottles of sodium chloride injection (U.S.P.) in the freezer of an ordinary refrigerator until ice cubes formed in a separate tray. During the procedure, a small bucket filled with crushed ice maintained the temperature in the bottle between $0^{\circ}$ and $-1^{\circ} \mathrm{C}$ ('hypothermic solution'). Analysis of our iced saline failed to reveal any increase in osmo- larity: $308 \mathrm{~m}$-osmol/1., consisting of $154 \mathrm{~m}$-equiv/l. sodium and $154 \mathrm{~m}$-equiv/l. chloride.

The patient was placed on a tilting table in the lateral position for lumbar tap with a 16 gauge spinal needle. Cerebrospinal fluid was allowed to flow out spontaneously until subambient pressure was produced. Mechanical withdrawal by syringe was specifically avoided to prevent aspiration of a nerve root or alteration of the needle tip within the subarachnoid space.

When the pain was unilateral, the patient remained with the affected side down. When the pain was bilateral or midline, the patient was placed in the prone position. The table was then tilted from $-60^{\circ}$ to $+60^{\circ}$, so that the appropriate area could be adequately irrigated.

A second 16 gauge needle was intermittently attached to a $12 \mathrm{ml}$. syringe for sterile aspiration of the hypothermic solution from the supply bottle. Repetitive injections were made as rapidly as possible to produce maximum cumulative cooling. Between injections, the spinal needle was left open; and the free back-flow allowed for continuous evaluation of needle placement as well as prevention of pressure build-up. After the final $12 \mathrm{ml}$. injection, back-flow was not permitted, and the needle was removed.

With the use only of local injection of lidocaine, there was severe experience of paraesthesias and fasciculations which interfered with rapid, repetitive irrigation and dissuaded patients from allowing subsequent cooling. A combination of fentanyl $0 \cdot 1-$ 
$0.2 \mathrm{mg}$ and droperidol $5-10 \mathrm{mg}$, followed by either sodium thiamylal $200-400 \mathrm{mg}$ or diazepam $20-40$ $\mathrm{mg}$, given intravenously just before the period of actual perfusion, was found to effectively reduce the discomfort while allowing evaluation of regional muscular contractions. At this light plane of anaesthesia, a total of $72 \mathrm{ml}$. iced saline could regularly be employed for lower quadrant pain, and $96 \mathrm{ml}$. for upper quadrant pain.

Fasciculations were limited to the period of actual cooling, while dysaesthesias continued in some of our patients for up to two hours and not infrequently required additional medication. Hypaesthesia was often noted in the affected area for periods of up to 48 hours. Headache and local discomfort from spinal tap occasionally necessitated administration of minor analgesics. Four patients with preexisting weakness of one extremity, secondary in two cases to known myelopathy and in two cases to suspected radiculopathy, experienced a transient increase in paresis for several days.

If the relief from pain was incomplete or less than 48 hours in duration, the entire procedure was repeated. If multiple areas of the body were involved with diffuse metastases, different levels were irrigated serially. In two patients the entire spinal cord was cooled, by three separate procedures in one and four separate procedures in the other.

\section{RESULTS}

Eighty subarachnoid irrigations with isotonic iced saline were performed on 50 patients: 33 with metastatic neoplasm, two with causalgia, four with neuralgia, five with low back pain persisting after laminectomy for herniated nucleus pulposus and six miscellaneous painful syndromes. In effect, 36 cases of lower quadrant pain and 18 cases of upper quadrant pain were treated during a one year period. Eleven patients with carcinoma obtained complete relief from intractable pain until death eight days to eight months later; 10 others had almost total relief for eight weeks before return of the original pain, and the remaining 12 had an equivocal result. Both patients with causalgia were free of symptoms for one month before the return of some discomfort in the affected area; neither showed preference for repeat spinal cooling. Essentially no relief was produced in the two patients with post-herpetic neuralgia, the one patient with post-thoracotomy intercostal neuralgia, or the one with post-herniorrhaphy ilioin- guinal neuralgia. Three patients with intractable low back pain after laminectomy and excision of $Z$ herniated nucleus pulposus have improved up to 12 months to date, while the other two had an equivocal result. The six miscellaneous painfulō syndromes included intractable buttock pain after coccygectomy, cervical radiculopathy per- $\frac{\widehat{S}}{\bar{S}}$ sisting after laminectomy and fusion, superior vena caval syndrome, ulcerative colitis, Buerger's. disease, and phantom limb; three obtained par- $\vec{\Rightarrow}$ tial relief, and three were unimproved in a one $\frac{\rho}{0}$ year follow-up.

No permanent sensory deficits, bladder dys- $\frac{\overline{\bar{N}}}{\bar{N}}$ functions, or other complications directly at- $\vec{\Phi}$ tributable to hypothermia have been observed in our series of 80 spinal cord coolings. Never- $\stackrel{\infty}{\overrightarrow{0}}$ theless, complications have been reported by other researchers, especially with the use of $\vec{\omega}$ hypertonic saline. Transient changes in blood pressure (Hitchcock, 1970) and cardiac arrhythmias (Hitchcock and McKean, 1968) have been attributed to direct stimulation of sympathetie nerve fibres by lumbar injection or of pari sympathetic nerve fibres by cisternal injectioß. O'Higgins, Padfield, and Clapp (1970) publishe을 an account of one complication of infarction 画 $\vec{c}$ the territory of the left middle cerebral arter 8. Hitchcock and McKean (1968) described ofe permanent cauda equina lesion.

\section{DISCUSSION}

Considerable evidence has been presented in favour of the concept that sense organs of the $\frac{0}{\bar{D}}$ skin with unmyelinated $C$ nerve fibres have widely varying functional characteristics (Christ- $\overrightarrow{\overrightarrow{0}}$ ensen and Perl, 1969; Bessou and Perl, 1970; Kumazawa, Perl, Burgess, and Whitehorn, 1971). In the mammal, a large proportion of such sensory units were excited by gentle mechanical stimulation and by transient cooling; $a_{-}$ second group responded best to small tempera- 3 . ture changes; and a third group had elevated thresholds for various or all stimuli. The intensity of the liminal stimulus and unique ability too provide signals marking the difference between $>$ innocuous, threatening, or damaging stimuli strongly indicated a nociceptive function for then high-threshold unmyelinated sensory units. The sensitization of polymodal nociceptors after noxious heat simulated certain kinds of hyper- $-\omega$ 
algesia. Furthermore, the pericornual cells of Rexed's lamina 1 could be fired antidromically by stimulation of the opposite anterior lateral funiculus in the cervical area, demonstrating the absence of internuncial cells and the dispensability of the modulation concept inherent in the gate control theory (Melzack and Wall, 1965).

Studies on the effects of temperature on neural conduction and the relative susceptibility of myelinated and unmyelinated nerve fibres have been contradictory. Von Euler (1947) suggested that unmyelinated $\mathrm{C}$ fibres were more susceptible to cooling than thickly myelinated fibres, which might even be stimulated. Lundberg (1948) reported conversely that the conduction of A fibres ceased at $7^{\circ}$ to $15^{\circ} \mathrm{C}$ in the cat, whereas conduction of splenic $\mathrm{C}$ fibres continued at $0^{\circ} \mathrm{C}$ in the cat and cow. Albin, White, and Locke (1967) confirmed the earlier observations of Brooks, Koizumi, and Malcolm (1955) and Suda, Koizumi, and Brooks (1957) that the action of cold on nerve conduction was opposite to that of local anaesthetic agents - the larger the cross-sectional diameter of the fibre, the more it was sensitive to hypothermia.

Laboratory research into the mechanism of relief of pain has been extensive but inconclusive; no animal model yet designed adequately simulates the complex human experience of intractable pain. The effects which have been documented are either transient phenomena or the results of extremes of temperature (DennyBrown et al., 1945) and osmolarity (Robertson, 1958; Jewett and King, 1971) not attained in the clinical situation. Only Tsubokawa (1969b) has made a comparative study of conduction block of A-delta and $\mathrm{C}$ fibres in the cat with appropriate cooling and without appreciable alteration of osmolarity: six hours after a 15 minute irrigation at spinal level L6-7 with $80 \mathrm{ml}$. iced saline (314 to $376 \mathrm{~m}$-osmol/1.), action potentials did not reappear in the A-delta or $\mathrm{C}$ fibres, while A-alpha and A-beta fibres were essentially unchanged; six days after two separate spinal coolings, $60 \%$ of the prepared animals failed to exhibit evoked potentials in the mesencephalic reticular formation or the centromedian nucleus of the thalamus after noxious stimulation of the sciatic nerve.

Nerve conduction studies were performed on a limited number of our patients. No change was demonstrated in the conduction velocity of normal peripheral nerves immediately after brief perfusion cooling of the subarachnoid space or at intervals up to six months. In one patient with radiculopathies involving spinal roots $C 6,7$, and 8 the right ulnar nerve tests were within normal limits, while the left ulnar nerve exhibited slowed conduction; after two separate irrigations, the left ulnar nerve remained electrically silent to testing.

Postmortem examination of one patient in our series failed to reveal any demonstrable neurolysis of the spinal cord or nerve roots. Mathews et al. (1970) also reported no histological changes in the medulla, spinal cord, or nerve roots of one patient who came to necropsy. Hitchcock (1967a), on the other hand, reported widespread vacuolization and pallor of myelin staining in one patient who came to necropsy.

Patients with successful relief of their intractable pain after spinal cord cooling still have appropriate perception of pin prick, skin pinching, and deep pressure and can identify correctly a hot or cold stimulus. These observations indicate that at least the crude reaction to noxious stimuli or temperature is present. Careful testing for threshold differences has not been carried out, yet the alleviation of intractable pain is in clear contrast to the apparent preservation of response to painful test stimuli.

Initial fasciculations and paraesthesias may be explained by transient activation of the larger $\mathrm{A}$ fibres in ventral and dorsal roots; subsequent hypaesthesia may be caused by inactivation of the sodium pump for a limited period of time. Sustained relief of pain may be attributed to cold trauma to a critical number of A-delta and C fibres or to pericornual cells and their ascending axons. Alternatively, gate modulation could be rendered inoperative by damage to a still undemonstrated anatomical substrate of interneurones. The exact role of spinal cord ischaemia secondary to vasospasm of the intrinsic vasculature remains undetermined (Meyer and Hunter, 1957; Douglas and Ritchie, 1957). Further experiments are necessary to ascertain whether the alleviation of pain is due to deactivation of peripheral fibres or to prolonged quiescence of central internuncial neurones.

The authors are indebted to Dr. Kuo, Department 
of Physical Medicine and Rehabilitation, Bronx Veterans Administration Hospital, who performed the nerve conduction studies.

\section{REFERENCES}

Albin, M. S., White, R. J., and Donald, D. E. (1961). Hypothermia of the spinal cord by perfusion of the subarachnoid space. Surgery Forum, 12, 188-189.

Albin, M. S., White, R. J., Locke, G. S., Massopust, L. C., Jr., and Kretchmer, H. E. (1967). Localized spinal cord hypothermia - anesthetic effects and application to spinal cord injury. Anesthesia and Analgesia, 46, 8-16.

Bessou, P., and Perl, E. R. (1969). Response of cutaneous sensory units with unmyelinated fibers to noxious stimuli. Journal of Neurophysiology, 32, 1025-1043.

Brooks, C. M., Koizumi, K., and Malcolm, J. L. (1955). Effects of changes in temperature on reactions of the spinal cord. Journal of Neurophysiology, 18, 205-216.

Christensen, B. N., and Perl, E. R. (1970). Spinal neurons specifically excited by noxious or thermal stimuli. Journal of Neurophysiology, 33, 293-307.

Collins, J. R., Juras, E. P., VanHouten, R. J., and Spruell, L. (1969). Intrathecal cold saline solution: a new approach to pain (evaluation). Anesthesia and Analgesia, 48, 816-823.

Denny-Brown, D., Adams, R. D., Brenner, C., and Doherty, M. M. (1945). The pathology of injury to nerve induced by cold. Journal of Neuropathology and Experimental Neurology, 4, 305-323.

Douglas, W. W., and Ritchie, J. M. (1957). Non-medullated fibres in the saphenous nerve which signals touch. Journal of Physiology, 139, 385-399.

Euler, C. von (1947). Selective responses to thermal stimulation of mammalian nerves. Acta Physiologica Scandinavica, 14, Suppl. 45.

Hitchcock, E. R. (1967a). Hypothermic subarachnoid irrigation for intractable pain. Lancet, 1, 1133-1135.

Hitchcock, E. R. (1967b). Hypothermic subarachnoid irrigation. Lancet, 1, 1330.

Hitchcock, E. R. (1969). Osmolytic neurolysis for intractable facial pain. Lancet, 1, 434-436.

Hitchcock, E. R. (1970). Hypothermic-saline subarachnoid irrigation. Lancet, 1, 843.

Hitchcock, E. R., and McKean, M. C. (1968). Electrocardio- graphic changes after intrathecal hypertonic saline solu- $\underline{Z}$ tion. Lancet, 2, 1083-1084.

Jewett, D. L., and King, J. S. (1971). Conduction block of monkey dorsal rootlets by water and hypertonic saline solutions. Experimental Neurology, 33, 225-237.

Kumazawa, T., Perl, E. R., Burgess, P. R., and Whitehorn D. (1971). Excitation of posteromarginal cells (lamina 1) in the monkey and their projection in lateral spinal tracts. Proceedings of the Twenty Fifth International Congress of $\triangle$ Physiology and Science, 9, 328.

Lundberg, A. (1948). Potassium and the differential thermosensitivity of membrane potential, spike and negative: afterpotential in mammalian A and C fibres. Acta Physiologica Scandinavica, 15, Suppl. 50.

Mathews, G. J., Ambruso, V. T., and Osterholm, J. L. (1970). Hypothermic, hyperosmolar saline irrigation of cisterna $\bar{O}$ magna: a new method for the relief of pain. Surgery $\bar{\omega}$ Forum, 21, 445-447.

Melzack, R., and Wall, P. D. (1965). Pain mechanisms: $a \stackrel{\mathbb{Q}}{2}$ new theory. Science, 150, 971-979.

Meyer, J. S., and Hunter, J. (1957). Effects of hypothermia on local blood flow and metabolism during cerebral $\vec{O}$ ischemia and hypoxia. Journal of Neurosurgery, 14, 210 227.

Negrin, J., Jr. (1970). The hypothermostat, an instrument to obtain local hypothermia of the brain or spinal cord. International Surgery, 54, 93-106.

Nicholson, M. F., and Roberts, F. W. (1968). Relief of painc by intrathecal injection of hypothermic saline. Medicals Journal of Australia, 1, 61-62.

O'Higgins, J. W., Padfield, A., and Clapp, H. (197\% Possible complication of hypothermic-saline subarachned injection. Lancet, 1, 567.

Robertson, J. D. (1958). Structural alterations in nerve fibets produced by hypotonic and hypertonic solutions. Jounial $l_{C}$ of Biophysics, Biochemistry, and Cytology, 4, 349-364.

Suda, 1., Koizumi, J., and Brooks, C. M. (1957). Analys of effects of hypothermia on central nervous system responses. American Journal of Physiology, 189, 373-38ळ 6

Tsubokawa, T. (1969a). Effect of subarachnoid perfusion $\lesssim f w$ frozen physiological saline on changes in action potential of the thalamus and dorsal root. Brain and Nerve, 21, 685 692.

Tsubokawa, T. (1969b). Method for relief of pain by injection of frozen physiological saline into the spinal subarachnoido space and clinical results. Brain and Nerve, 21, 693-697. 